\title{
BMJ Open Quality Ethical considerations in quality improvement: key questions and a practical guide
}

\author{
David Francis Hunt (D , ${ }^{1,2}$ Michael Dunn, ${ }^{3}$ Guy Harrison, ${ }^{4}$ Jill Bailey,6
}

To cite: Hunt DF, Dunn M, Harrison G, et al. Ethical considerations in quality improvement: key questions and a practical guide. BMJ Open Quality 2021;10:e01497. doi:10.1136/ bmjoq-2021-001497

Received 17 March 2021 Accepted 25 July 2021

A Check for updates

(c) Author(s) (or their employer(s)) 2021. Re-use permitted under CC BY-NC. No commercial re-use. See rights and permissions. Published by BMJ.

${ }^{1}$ Department of Experimental Psychology, University of Oxford Medical Sciences Division, Oxford, UK

${ }^{2} 0 x f o r d$ Healthcare Improvement Centre, Oxford Health NHS Foundation Trust, Oxford, UK

${ }^{3}$ The Ethox Centre and

Wellcome Centre for Ethics and Humanities, University of Oxford, Oxford, UK

${ }^{4}$ Department of Spiritual and Pastoral Care, 0xford Health NHS Foundation Trust, Oxford, UK

${ }^{5}$ Faculty of Health and Life Sciences, Oxford Brookes University, Oxford, UK

${ }^{6}$ Coventry and Warwickshire Partnership NHS Trust, Coventry, UK

Correspondence to Dr David Francis Hunt; david.hunt@psy.ox.ac.uk

\section{ABSTRACT}

Quality improvement (QI) provides a rigorous and innovative approach to improving patient's lives in the healthcare system. Still, it can pose challenges in understanding what ethical considerations apply to some projects to minimise the possibility of patient harm or prevent other ethical wrongs and potential staff burden. While many commentaries discuss the extent to which QI ethics should match research ethics, there is minimal literature regarding what $\mathrm{QI}$ project teams should do when considering ethics at the planning stage. This paper provides a practical walkthrough of some of the ethical considerations across the breadth of QI projects, starting from some of the key questions when planning a QI project and a guide for the different ethical considerations that may apply.

\section{INTRODUCTION}

The Health Foundation describes quality improvement (QI) as the systematic approach that uses techniques to improve healthcare quality. ${ }^{1}$ QI projects vary in scale and can include individual-level, group-level and organisational-level tests of change that can involve large groups of staff, patients and their carers/families in one or across various healthcare settings. Following the Institute of Healthcare Improvement's model involves a diagnostic stage to establish what the team is trying to accomplish, whether an improvement is possible, and what changes may result in an improvement. Following this diagnostic stage, one or more change ideas are tested using a Plan, Do, Study, Act cycle through an iterative process. ${ }^{2}$ It is important to note that other QI approaches are utilised in healthcare, but this paper adopted the IHI model for QI to develop this guidance.

Although QI can create significant benefits, it can also shape patients' care in ways that raise ethical questions concerning respect, dignity, harm and justice. In particular, QI in healthcare can involve changes in care delivery and have unanticipated adverse outcomes and cause harm to patients. ${ }^{3}$ These harms vary from time spent completing unnecessary surveys to physical and psychological harm.
Relatedly, the risk of changing care delivery must be measured on a risk/benefit basis and weighed against the increased risk to patients compared with usual care. ${ }^{4}$ Poorly justified and delivered QI projects that are unlikely to yield positive results may not be ethically justified when considering the burden to patients. ${ }^{5}$ As such, careful consideration needs to be paid to ensure that QI projects are delivered in fair and equitable ways and whether they might infringe on patients' dignity or autonomy. Similarly, family/carer involvement, especially those who may be directly involved in care delivery, are at risk of potential harm from the consequences associated with conducting a poorly conceived and/or poorly delivered QI project.

As well as the potential patient harm and burden that may arise from a QI project, potential staff harm and burden also requires consideration. Typically, staff lead QI projects in their locality or service and are responsible for their completion. As such, they may accrue operational and emotional burdens from carrying out a project that detracts from direct patient contact or carries a perceived element of controlled or even uncontrolled risk.

Despite the potential associated benefits and costs of QI, there is much confusion over identifying the appropriate ethical considerations for QI and how to address these in a justifiable and practically feasible manner. In general, QI projects do not typically require the same ethical approval as research projects. $^{6}$ Any ethical rules in place for the conduct of QI activities are often unclear. ${ }^{4}$ Furthermore, some QI projects do not require patient consent and clinician participation is not always voluntary. ${ }^{7}$ These challenges are further compounded by the wide variation in QI projects and the extent to which ethical considerations must be applied.

The extent to which ethical oversights need to be established has led to many commentaries on the differences and similarities 
between ethics in QI and research. ${ }^{6-10}$ This has been stimulated further by recent work in research ethics that has scrutinised the ethical obligations that arise within a 'learning health system'. ${ }^{11} 12$ The consensus is that QI activities cut across the established demarcation between clinical ethics and research ethics and that distinctive ethical requirements arise for implementing QI projects. While well-established ethical guidance is available for research activities, there is a paucity of information on how to conduct QI projects in an ethically defensible manner, despite the emergence of detailed, practical guidance for implementation research more generally. ${ }^{13}$ The Healthcare Quality Improvement Partnership produced ethical guidance designed to differentiate between research, QI and clinical audit, and how these projects should be reviewed and monitored. ${ }^{14}$ While this report provided helpful guidance for identifying some of the possible ethical issues, it does not provide clear, practical guidance that focuses on the day-to-day tasks associated with carrying out various stages of an ethical QI project and the ethical judgements that need to be made within these stages.

A lack of clear ethical guidance in a QI project can lead to ethical considerations being side-lined, ignored or addressed in a cursory manner, potentially giving rise to harms or the undermining of trust and accountability in healthcare settings. ${ }^{15}$ Also, as healthcare staff often work in high-pressured environments, many might be tempted to adopt a pragmatic 'get it done' approach to QI projects and unintentionally miss critical ethical considerations. Furthermore, this lack of clear guidance for ethics in QI can lead to teams modifying projects to fit within a QI context to circumvent research ethics review requirements that often take a considerable amount of time. This practice is questionable because QI projects are not absent of ethical issues that ought to be attended to prior to being implemented.

This paper sets out a practical guide and 'walkthrough', tailored explicitly for ethical considerations in QI projects. This guidance was borne out of our collective experiential knowledge of supporting and leading QI projects in a mental healthcare setting. As such, the key questions and guidance are a composite of the experience of both managing ethical challenges across many projects with teams and QI project oversight meetings. First, we held a series of meetings to identify key themes, successes and lessons learnt from our experiences. Next, we selected QI project examples that would best illustrate how to apply our suggested guidance. Finally, we discussed the guidance with different QI leads and QI researchers locally to ensure that the key questions and guidance were fit for purpose and revised when necessary.

Unlike previous guidance, this paper provides a granularity of detail to guide novice and experienced QI teams through addressing the ethical dimensions of their projects, with due consideration for all parties involved. Furthermore, we intentionally designed the guidance to be as generalisable as possible and applicable, irrespective of the particular person implementing the QI project, whether this is a front-line clinician, executive leadership, or QI leads. Finally, the guidance is grounded in QI projects to maximise its usefulness to all involved in this work.

First, we set out a list of key questions to assist in identifying the range of ethical considerations that could present themselves when conducting a QI project. Next, we provid practical guidance that sets out the typical stages in a QI project and associated actions. Finally, we provide different QI examples and how these ethical considerations apply alongside the QI activities. Following this, we pull out some of the key themes and discuss their importance in a QI project context.

\section{KEY QUESTIONS WHEN CONDUCTING QI PROJECTS}

As a QI project can vary considerably in the extent to which ethical considerations apply, teams must understand how their projects raise ethical considerations that they need to attend to, throughout the project, from planning through to implementation. These questions should consider the categories of patient and family/ carer, staff involvement and burden, mental capacity and consent, accessing data, and whether there is a possibility of harm to any of the parties involved in the project. Box 1 provides some of the possible key questions when planning a QI project.

\section{KEY ETHICAL CONSIDERATIONS WHEN CONDUCTING QI PROJECTS}

When planning the breadth and extent to which ethical considerations apply to any given QI project, the next step is to consider the list of actions required at every project stage. Box 2 provides suggested actions throughout all project stages, focusing on what staff should do when conducting QI projects and incorporating them into their daily activities (see box 2). These align with the questions posed in box 1 .

\section{APPLICATION OF ETHICAL CONSIDERATIONS TO QI PROJECT EXAMPLES}

The extent to which ethical considerations apply to different QI projects varies drastically from project to project. For some projects, it is a matter of changing an administrative process that can impact patient outcomes further down the line. At the other extreme, it can involve exploring sensitive topics that may cause distress and harm to those involved. Table 1 provides examples of the different types of projects and what ethical considerations may be applied.

Boxes 1 and 2, table 1 illuminate the numerous ethical considerations throughout any QI project, from inception to completion and beyond. The following section elaborates on some of the more pertinent ethical considerations in QI projects that pose particular complexities and are worthy of further reflection. 


\section{Box 1 Questions when starting a QI project}

\section{Scope of the project}

- Is the project relating to the exercise or organisation of front-line healthcare provision or supporting healthcare duties?

- Are all relevant staff involved in scoping the project?

\section{Patient and family/carer engagement}

- Have you considered whether the method for engagement is suitable for all the project participants, given the overarching aim or specific QI project's objectives?

- Do the patients and/or family members and carers understand the purpose of the QI project and their role in the project?

- Will there be any qualitative or quantitative data collection directly from patients and/or family members and carers?

If so, how will these data be collected and by whom?

\section{Staff engagement}

- Is there a registered team member to provide oversight of the project and adhere to any local governance requirements?

- Are all relevant staff involved in all stages of the project?

- Are staff provided with appropriate time, support and training to lead or support a QI project?

- Are the staff members' views or experiences being collected, documented and analysed/considered as part of the project?

- Have all concerns about the project and its feasibility been addressed? (These may include staffing capacity and capability concerns and concerns around the relative risk related to a proposed test of change).

\section{Informed Consent}

- If the project directly involves patients and/or family members and carers, who will facilitate the provision of informed consent?

-Who will assess mental capacity to give consent?

Will consent/agreement be required from the lead clinician?

- How will consent be documented? By whom?

- If the project directly involves collecting data from staff, are staff fully informed about the project and what it will involve for them?

\section{Accessing patient data}

- Will the project require accessing identifiable patient information?

- Are relevant information governance procedures followed or advice needed?

$>$ Does patient participation need to be recorded in their record? Who will take responsibility for this?

- How will the data be curated and analysed?

\section{Possible distress and harm}

- Is there any possibility of causing physical and/or psychological harm?

- Is there a risk of causing harm to the patient or vicarious trauma to the researcher or staff involved?

Was the involvement itself, traumatic? If so, is there a plan to support the patients, family members, carers or staff members?

\section{STAFF, PATIENT AND FAMILY/CARER ENGAGEMENT}

Patient, family/carer and staff views are essential to conducting ethical and safe QI projects that maximise the chances of improving healthcare. Incorporating patient and staff views ensures that any QI work is fit for purpose and patient-centred, reflecting their needs, and ensuring that their involvement is 'with' or 'by' patients and staff, rather than 'to' or 'for' them. ${ }^{16} \mathrm{QI}$ projects give
Box 2 Guidance for addressing ethical considerations in quality improvement (QII) projects

\section{Stage and suggested actions}

The impetus for the project

- Attend a series of meetings with relevant gatekeepers and stakeholders to discuss the motivations for starting the project and the anticipated benefits to improve healthcare delivery.

- Where possible, include an evidence base (literature or data or both) to discuss the rationale behind why this work is important.

- Identify and explore the benefits and opportunities for QI capability building.

- Establish senior leader buy-in to protect time for staff to lead QI projects, if necessary.

Validity and value of the project

- Scope the evidence base for the tests of change considered.

- Ensure that the tests of change align with the project aims and that all those involved understand and contribute (where appropriate) to the diagnostic stage of the project.

- Ensure that measures are valid, reliable and have an evidence base.

- Establish a measurement strategy capable of reliably evaluating whether tests of changes are successful in improving patient care.

- Designate a QI lead (typically a clinician) to ensure that the project is viable and considered.

- Ensure that the project adheres to any local ethical oversights.

\section{Staff, patient and family/carer engagement}

- Engage staff, patient and family/carer viewpoints throughout the project to ensure that their insights inform whether the project is feasible and remains patient-centred (where appropriate).

- Empower and engage relevant staff taking ownership and leading the project.

- Remind all those involved about the importance of their engagement, the expectations regarding their involvement, and ensure they are credited throughout the project and beyond.

- Ensure staff are broadly on board with the project's benefits and that they are fully informed about the impact on their day-to-day duties (eg, any additional time that may impact the amount of patient care provided).

- Consider the methodological approach when collecting views and whether they reflect all viewpoints and maximise their contributions.

- Ensure that accessible language used is understandable to the intended audience. Possibly pilot for all materials associated with a project (eg, questionnaires).

- Ensure that the questions will give insight into current practice.

- For Ql, the questions should focus on improvement (ie, knowledge, perceptions and suggestions) and focus less on personal experience unless collecting data on direct experience is judged as critical to evaluating any improvement.

- Ensure that when collecting the views of patients and families/ carers that it recognises the relevant sensitivities and vulnerabilities-start with a lighter opening question to serve as an ice breaker, gradually deepen the questions, and check if the patient is ok to return to the ward/their daily life.

- Ensure that engaging with patients is considered with minimal disruption to their current routine.

- Ensure flexibility when engaging with staff (eg, establishing protected time for their Ql activities).

- Provide a comfortable and neutral space that allows staff and/or patients to have open and candid discussions.

Continued 


\section{Box 2 Continued}

- Be mindful of environmental considerations for more confidential and/or sensitive discussions.

- Be mindful of body posture and non-verbal cues when engaging staff, patients and family/carers about their views.

- Engage collaborative discussions in group settings and be inclusive but focused on integrating ideas into a project.

\section{Informed consent}

$\checkmark$ Discuss patient recruitment with staff to be aware of any issues that may arise.

- Consult established guidance on producing information sheets and/ or provide consent forms in the research ethics context.

- Patients and relevant family/carers should be prebriefed before the project takes place and understand the nature of the aims.

- Gain informed consent before patient involvement.

- Ensure that they understand what is happening and fully understand their right to withdraw and ask questions.

- Any relevant parent/carer needs to receive communication about the projects, including a right to object or ask any questions.

- Obtain consent from the clinical lead to indicate who may be willing to participate and who may not be suitable.

- Collect informed consent for staff and families/carers, when applicable.

- Create an appropriate procedure to anonymise the identity of each patient when necessary.

- Record consent and participation in patients' records, where appropriate.

\section{Patient information and data collection}

- Collate the required information for measuring baseline and effectiveness of tests of change.

- Wherever possible, collect from existing data sources and avoid creating an additional burden on the staff's time.

- Ensure that those accessing staff, patient and family/carer identifiable information have received the necessary training.

- Access any data using appropriate computer equipment (approved equipment or via agreed remote desktop access).

- Ensure that software used to explore/analyse data is safe and free from possible data breaches.

- Store all data in a secure location (eg, a secure drive within the organisation).

- Ensure that all data curation, handling and dissemination align with local data policies and are compliant with the Data Protection Act (2018).

\section{Welfare and safeguarding}

- Consider possible harm and burdens that might accrue to all those involved at all project stages.

- Ensure a risk assessment is in place if the staff, patients or family/ carers involved become distressed or provide a disclosure during any part of the project (eg, collecting views, qualitative evaluation).

> Ensure that all parties involved know procedures to signpost individuals to relevant services (eg, a web link or print out to agreed internal and external services).

- All staff involved must receive safeguard training and understand local safeguarding policies.

- Ensure appropriate professional indemnity is in place, particularly when QI teams engage with services outside their immediate work environment.

\section{Box 2 Continued}

When collecting patient and/or family/carer views, consider including a staff member to act as a familiar face and comply with the safeguarding requirements above.

- There should be wellness checks with all parties involved when entering any potentially sensitive or difficult activity in a project.

- Record all sensitive activities with direct patient involvement on the electronic patient record.

- For sensitive discussions, interviews and focus groups, staff should be debriefed with an appropriate colleague (eg, a supervisor or manager) as part of the wellness check and to discuss the outcomes.

rise to wide-ranging ethical considerations that concern all parties involved, as they typically experiment with changes in healthcare delivery that may be embedded into practice. Below, we discuss requirements to engage the different groups and how this is important in propagating ethical practice.

\section{Staff engagement}

QI projects are unique from most research in two aspects. First, staff lead many of the QI projects as an integral part of their day. Second, any staff member at any level of the organisation can lead a QI project. Across all QI projects, healthcare organisations should also consider designating a senior leader to oversee whether QI projects are viable and maintain ethical monitoring, a point made in previous guidance. ${ }^{14}$ Organisations may designate a leader in each service or directorate, create a dedicated team or assign these responsibilities to an existing team.

The QI leadership teams typically support these teams in simultaneously learning (if they are new to QI) and implementing a project in their service. This approach leads to two significant ethical considerations regarding the role of the staff leading the QI project. First, the extent to which their time is justifiable, especially for those who incur additional tasks that detract from patient care, typically in their current role. ${ }^{17}$ The second is a possible emotional burden for staff conducting QI projects with an element of perceived risk (eg, reducing the length of stay that could increase readmission if the project is unsuccessful). These considerations are essential in all QI projects, especially when projects do not have total staff investment when starting a project. ${ }^{18}$

Besides direct staff engagement, their engagement as stakeholders interested in the project and gatekeepers for their patient populations are equally important. Gatekeeper examples can include a broad range of audiences, such as carers and families of those that do not have mental capacity, the nursing or clinical lead (eg, Ward Manager or Matron), members of any advocacy team, and clinical medical leads (eg, consultant) or regulators (eg, specific boards with regulatory responsibilities). Decisions around allowing access to their group(s) should broadly follow three critical criteria: 
Table 1 Application of ethical considerations to QI project examples

\section{Project 1: low risk to patients and staff}

Improving the efficiency of invoicing processes for the procurement of agency nursing staff

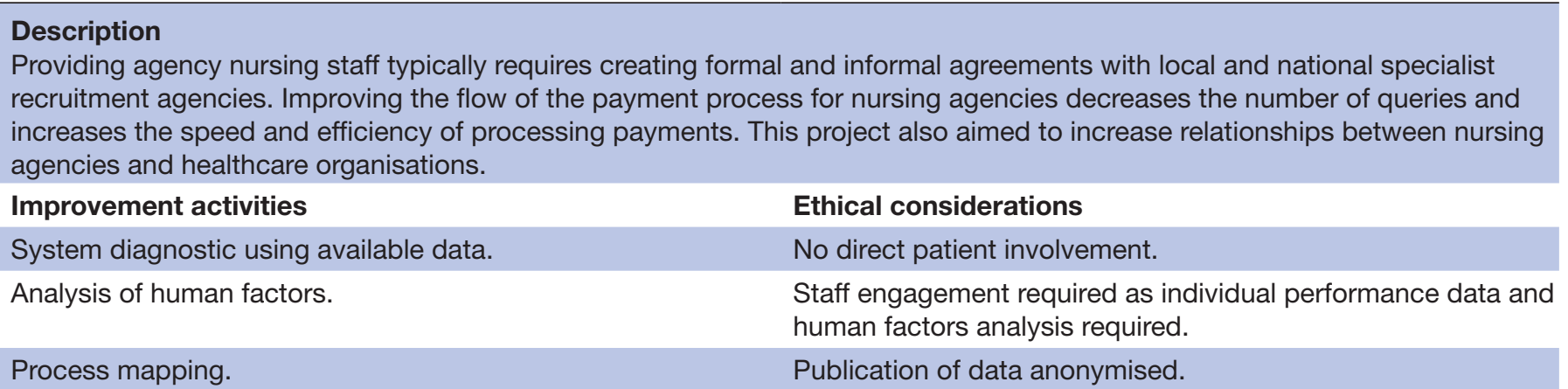

Tests of change included: (1) Ensured that all managers

sourced agency workers through the staffing solutions team;

(2) All shifts were added to the appropriate system; (3) Raised

awareness to all appropriate managers that all agency shifts

should be adjusted and finalised.

End to end process re-engineering.

Project 2: potential moderate risk to patients and staff

Well-being Wednesdays: nurse-led clinic for improving physical healthcare in a general adolescent inpatient unit ${ }^{23}$

\section{Description}

Young people with mental illness are at high risk of physical health complications. The reason for this includes not seeking treatment for physical health issues, engaging in unhealthy behaviours (eg, drug use, smoking, poor sleep, poor diet and sedentary behaviour), and side effects associated with certain medication for psychiatric treatment. The project aim was to establish a well-being clinic to improve the efficiency and quality of physical healthcare and health promotion offered to young people.

\section{Improvement activities}

Carry out a system diagnostic using patient data via clinical audit for baseline measures, multidisciplinary staff engagement and patient engagement in codesigning the Well-being Wednesday Clinic.

Tests of change included: (1) Establishment of a well-being Wednesday physical health clinic with associated physical parameters and measures; (2) Modified the clinic, established a well-being board and created specific physical health plans and blood test protocols; (3) Made links to other associated services (eg, school nurses, sexual health) and further specific training (eg, phlebotomy, ECG and vaccinations) to enhance the offerings. A second nurse was appointed to lead the clinic.

\section{Ethical considerations}

Balance patient involvement in codesign when exploring feasibility against possible safeguarding concerns, particularly when patients are acutely unwell and detained under the Mental Health Act (MHA) 1983.

Staff engagement to open discussions around capacity, capability and confidence in additional professional duties associated with physical health.

Compliant with the Data Protection Act (2018).

Promote the benefits of additional checks to patients and empower their participation in their own physical health. Ensure buy-in from the team regarding the importance of physical health measures.

Provide training and related policies to improve the competence and confidence of staff.

Feedback to ensure the clinic is 'fit for purpose' and avoid duplication of administrative tasks.

Increased documentation detracts from direct care.

Involve related services to ensure patient care being immediate staff's professional boundaries.

\section{Project 3: Potential high risk to patients, families, public and staff}

Absconding: Reducing failure to return in adult mental health wards ${ }^{24}$

Description
When patients fail to return from Section 17 (MHA, 1983) leave or informal time away from the ward as part of their planned
recovery process, it may result in negative consequences to patients, family and staff. Tests of change to promote positive
risk-taking included a signing in and out book, individual safe leave forms, patient information leaflets, appointment cards with
agreed return times and contact numbers, intentional rounding, and visual procedural guidance for staff. These tests of change
increased the rates of patients returning from leave at the agreed time.

Continued 
Table 1 Continued

Project 3: Potential high risk to patients, families, public and staff

Absconding: Reducing failure to return in adult mental health wards ${ }^{24}$

\section{Improvement activities}

Carry out a system diagnostic using patient data, patient experiences, ward observations, process mapping with the staff team.

\section{Ethical considerations}

Direct patient involvement in co-design when patients are acutely unwell and detained under the MHA 1983. Mental Capacity Act implications for practice, including issues of capacity to consent to participate. Recording of participation and consent.

Staff engagement to open discussions about ward culture regarding leave procedures standards and the importance of managing and negotiating leave well and collecting data in practice. Recording of participation and consent.

Ensure family engagement in the re-designed process, including enhanced safety checks with family and possible impact.

Compliant with the Data Protection Act (2018).

Tests of change included: (1) Signing in and out record; (1) Individually completed safe leave forms for discussion at ward rounds; (2) Improved patient information leaflets; (3) Appointment cards to prompt return to the ward; (4) Intentional rounding to assertively manage the leave process every hour.
Balance of autonomy versus security in the context of individualised risk assessment and the advocacy-paternalism continuum. Clearly communicate the new procedure with compassion. Tensions between staff and patients may arise during early implementation.

The organisational policy was rewritten to clarify the definition of missing and absent without official leave to protect staff and patients. Agreement between Police and staff to ensure a standard response time and action for every patient who fails to return. Standardised practice increases the time for direct care.

Increased documentation time detracts from direct care.

Use of plain English and all necessary languages in the information.

Provided unidentifiable 'appointment cards' in case of loss during the leave period.

Intentional rounding may be perceived as intrusive or irritating for patients. It, therefore, requires a sensitive approach.

QI, quality improvement.

1. Minimising the risk of harm and maximising benefit from the QI project-both in their immediate service, relevant linked services and the wider community.

2. Ensure that there are likely to be distinct benefits from the QI that align with organisational aims and/or current priorities.

3. There will be a reasonable level of resource and support, depending on their commitment and what the QI project entails.

A valuable approach to staff engagement is collaborating with them throughout the project to design and carry out activities to achieve the aims. Relatedly, particular leadership behaviours encourage staff collaboration and engagement. These behaviours include compassionate and inclusive leadership that encourages psychologically safe practice and candid discourse at all stages of a QI project. ${ }^{19-21}$ Both staff collaboration and leadership behaviours are vital for multiple reasons.
1. Staff input is especially crucial for QI project focus, diagnosis and suitability of a QI project that positively influences patient treatment and experience.

2. Leadership engagement will directly impact whether projects are embedded in routine practice and sustained.

3. Leadership and staff collaboration can find opportunities to decrease work burden in related aspects of patient care (eg, increased efficacy from reducing duplication).

4. Leadership can highlight safeguards regarding staff involvement in a project (eg, burden) and its impact on their patient population (eg, staff cover and protected time).

From a cultural perspective, collaboration with staff empowers them to shift from passive recipients to active contributors and QI project leaders. Furthermore, successful collaborations provide opportunities to gain 
their insights through open dialogue and balancing these with data-informed and research-informed approaches to maximise QI projects' effectiveness. Providing support to the staff who lead a QI project offers them an opportunity to simultaneously learn and implement QI practice, leading to individual and organisational capability building.

\section{Patient engagement}

The primary purpose of QI projects is to provide changes that result in increased quality of patient care, focusing on patients' lived experience in living with particular conditions and the impact of received care. Seeking patient engagement offers the opportunity to illuminate the value of a project to them, providing insights provide opportunities for codesigning and redesigning QI projects. Furthermore, patient involvement keeps the patient at the centre of QI efforts.

Despite the benefits, patient engagement is complex and has many ethical challenges. Without proper consideration, patriarchal approaches to patient engagement can lead to negative consequences, including leaving them disempowered and damaging therapeutic relationships. ${ }^{22}$ Also, the extent to which patients can engage may vary based on their current health status and capacities. QI projects should provide a flexible approach to patient engagement that reflects their varying degrees of capacity. Examples of approaches include patient attendance at project meetings, one-off discussions or repeated discussions to explore their views throughout a project, and the extent to which they engage in the codesign of various aspects of the project (eg, preparation of material, inputting on change ideas).

Successful patient engagement requires organisational support and collaboration with staff to ensure safe and successful input from patients. This collective effort provides opportunities to ensure that the approach is 'fit for purpose' and will empower patients to contribute. As discussed in box 1, patients should understand the importance of their input and feel safe to speak up even if they disagree with a suggested course of action. They should also understand their role and associated expectations and receive appropriate credit for their contributions.

\section{Family/carer engagement}

Patient care can often be influenced and directly supported by family and/or carer engagement in formal and informal settings. Families and carers also provide an opportunity to advocate and bridge the gap between patients and the healthcare system, particularly when patients may not have mental capacity. Furthermore, families and carers offer lived experience and experiential knowledge to support patients and possibly inform changes in formal healthcare practice.

Like patient engagement, family/carer engagement considerations must be weighed against numerous ethical considerations-for example, the balance between the value of their insights and whether they provide effective patient care. Also, considerations regarding patientcentred care and issues relating to confidentiality (eg, balancing family/carer engagement alongside patient engagement and whether this reflects the patient's wishes). Like patient engagement, staff should understand the role of family/carer engagement to ensure they feel empowered to contribute and incorporate their involvement wherever appropriate. Furthermore, families and carers should realise the importance of their contributions, understand expectations and credit them throughout the project.

\section{SAFEGUARDING AND WELFARE}

Welfare and safeguarding are paramount to all concerned when conducting a QI project and should be the priority at all stages. This can include (but is not limited to) handling patient and staff data, issues relating to the privacy and confidentiality of patient and staff identities, and the adverse effects resulting from the project.

A sensible starting point for any QI project is to devise a protocol for handling safeguarding issues and ensure that those conducting QI projects are suitably trained. In particular, at points of the project where the risk of a safeguarding issue is much greater. For example, exploring experiences based on sensitive topics or possibly resurfacing previous trauma or uncovering a new safeguarding disclosure. Any drafted protocol must align with current safeguarding practices and should be codesigned with a designated safeguarding representative. All parties involved in the project should be familiar with this process to provide seamless support should a safeguarding issue arise. All those conducting QI should receive relevant safeguarding training and refreshers as part of their mandated training. This is particularly important for organisations that employ QI Leads. For individual projects, all those involved in QI projects should be aware of any local safeguarding procedures.

As well as the immediate safeguarding issues when conducting a QI project with patient engagement, it is also vital to consider any possible long-term effects. Any protocol should provide welfare checks and subsequent support procedures for all those involved. In particular, for sensitive topics, a mandatory supportive review should be available to all those involved in the project. A sufficiently trained person should facilitate the review and be removed from the project itself. If any further distress or issues arise, all those involved should be signposted/ referred to any other relevant professional services or internal services. Practical approaches include recording the extent to which patients were involved in the project and associated documents.

\section{LIMITATIONS}

The proposed guidance has several limitations worth considering. First, the evidential basis for this guidance is mainly grounded in one mental health trust and the authors' experiences. Therefore, differences in ethical 
oversight may influence the usefulness of the proposed guidance. For example, some healthcare organisations may have pre-existing ethical governance frameworks in place or have already established processes with internal research and development teams or ethics committees. Second, the current QI capacity and capabilities between healthcare organisations may also vary drastically. For example, some healthcare organisations may have dedicated QI teams. Some may have external support with no dedicated teams, and others may have QI integrated into other teams, such as audit teams and research and development teams. Due to the diverse nature of QI projects (as illustrated in table 1) and QI capabilities between healthcare organisations, this paper is designed for teams to shape, reflect and use this guidance at their discretion depending on what applies to their QI project rather than being a prescriptive framework.

\section{CONCLUSION}

The extent to which QI projects require ethical approval varies considerably and, in some cases, align substantially with similar research projects. It is sometimes difficult for those in the QI field to know the extent to which ethical considerations are required, and this ambiguity can cause confusion and potential harm. This paper has shown how QI projects give rise to ethical considerations that demand attention, regardless of whether $\mathrm{QI}$ and research ethics ought to be treated as distinctive or overlapping activities. There are residual questions about whether QI should be subject to ethical oversight and ethics review processes, as is the case in research activities. However, we leave these questions for future analysis.

Instead, this paper sought to provide task-oriented guidance accessible to busy healthcare staff in their day-to-day duties, distinctively supplementing previous guidance documents. It aids those conducting QI projects, shifting mindsets from a 'get it done' mentality to a more considered ethical QI practice approach. Furthermore, we sought to explore the ethical considerations for conducting QI projects for all involved, including carers/patients and staff who support and lead these projects, focusing on the importance of engagement. In the first instance, those undertaking QI projects need to think proactively about the ethical considerations in their work before implementing QI tests of change. The key questions outlined aim to help guide what type of ethical considerations are essential for each project. Next, we outline practical guidance for various stages in a QI project and associated actions. Finally, we discuss QI project examples and the ethical considerations applied to each project.

Incorporating the key questions and guidance provides QI teams (and researchers in QI) to ensure considered, proportionate, and ethical practice in future QI projects, given the impact these projects (and their evaluation) can have on patient's healthcare staff.

Twitter David Francis Hunt @dfhunt1981
Acknowledgements We would like to thank the teams at Oxford Healthcare Improvement Centre at the Oxford Health NHS Foundation Trust, and the Risk and Safety Group at the Department of Experimental Psychology at the University of Oxford for their contributions and feedback on this manuscript.

Contributors All authors contributed to idea development. DFH and JB drafted the initial manuscript. MD and GH contributed to refining the manuscript.

Funding The authors have not declared a specific grant for this research from any funding agency in the public, commercial or not-for-profit sectors.

Competing interests None declared.

Patient and public involvement Patients and/or the public were not involved in the design, or conduct, or reporting, or dissemination plans of this research.

Patient consent for publication Not required.

Provenance and peer review Not commissioned; externally peer reviewed.

Open access This is an open access article distributed in accordance with the Creative Commons Attribution Non Commercial (CC BY-NC 4.0) license, which permits others to distribute, remix, adapt, build upon this work non-commercially, and license their derivative works on different terms, provided the original work is properly cited, appropriate credit is given, any changes made indicated, and the use is non-commercial. See: http://creativecommons.org/licenses/by-nc/4.0/.

ORCID iD

David Francis Hunt http://orcid.org/0000-0002-8802-7368

\section{REFERENCES}

1 Health Foundation (Great Britain). Quality improvement made simple: what everyone should know about healthcare quality improvement: quick guide. Health Foundation, 2013.

2 Langley GJ, Moen RD, Nolan KM. The improvement guide: a practical approach to enhancing organizational performance. John Wiley \& Sons, 2009.

3 Baily MA, Bottrell MM, Lynn J, et al. Special report: the ethics of using Qi methods to improve health care quality and safety. Hastings Cent Rep 2006;36:S1-40.

4 Lynn J, Baily MA, Bottrell M, et al. The ethics of using quality improvement methods in health care. Ann Intern Med 2007; 146:666-73.

5 Bellin E, Dubler NN. The quality improvement-research divide and the need for external oversight. Am J Public Health 2001;91:1512-7.

6 Stiegler MP, Tung A. Is it quality improvement or is it research?: Ethical and regulatory considerations. Anesth Analg 2017;125:342-4.

7 Horsfall J, Cleary M. Mental health quality improvement: what about ethics? Int J Ment Health Nurs 2002;11:40-6.

8 Cribb A, Entwistle V, Mitchell P. What does 'quality' add? Towards an ethics of healthcare improvement. J Med Ethics 2020;46:118-22.

9 Fiscella K, Tobin JN, Carroll JK, et al. Ethical oversight in quality improvement and quality improvement research: new approaches to promote a learning health care system. BMC Med Ethics 2015;16:1-6.

10 Flaming D, Barrett-Smith L, Brown N, et al. "Ethics? But it's only quality improvement!". Healthc Q 2009;12:50-5.

11 Faden RR, Kass NE, Goodman SN, et al. An ethics framework for a learning health care system: a departure from traditional research ethics and clinical ethics. Hastings Cent Rep 2013;Spec No:S16-27.

12 Kelley M, James C, Alessi Kraft S, et al. Patient perspectives on the learning health system: the importance of trust and shared decision making. Am J Bioeth 2015;15:4-17.

13 Peters DH, Tran NT, Adam T. Implementation research in health: a practical guide. World Health Organization, 2013.

14 Dixon N. Guide to managing ethical issues in quality improvement or clinical audit projects. London, UK: Healthcare Quality Improvement Partnership, 2017.

15 Kass NE, Faden RR. Ethics and learning health care: the essential roles of engagement, transparency, and accountability. Learn Health Syst 2018;2:e10066.

16 Hayes H, Buckland S, Tarpey M. Briefing notes for researchers: public involvement in NHS, public health and social care research. Eastleigh: INVOLVE, 2012.

17 Draper DA, Felland LE, Liebhaber A, et al. The role of nurses in hospital quality improvement. Res Brief 2008:1-8.

18 Disch J, Sinioris M. The quality burden. Nurs Clin North Am 2012;47:395-405.

19 Hunt DF, Bailey J, Lennox BR, et al. Enhancing psychological safety in mental health services. Int J Ment Health Syst 2021;15:1-18. 
20 West MA, Chowla R. Compassionate leadership for compassionate health care. Compassion 2017;21:237-57.

21 Smith ME, Finks JF. Collaborative Quality Improvement. In: Health services research. Cham: Springer, 2020: 137-53.

22 Dent M, Pahor M. Patient involvement in Europe--a comparative framework. J Health Organ Manag 2015;29:546-55.
23 Mellor C, Blackman J, Barker E, et al. Wellbeing Wednesdays: nurseled clinic for improving physical health care in a general adolescent inpatient unit. Br J Nurs 2020;29:230-5.

24 Bailey J, Page B, Ndimande N, et al. Absconding: reducing failure to return in adult mental health wards. BMJ Qual Improv Rep 2016;5:u2 09837-w5117. 